\title{
Photonic Metamaterials: Magnetism at Optical Frequencies
}

\author{
Stefan Linden, Christian Enkrich, Gunnar Dolling, Matthias W. Klein, Jiangfeng Zhou, Thomas Koschny, \\ Costas M. Soukoulis, Sven Burger, Frank Schmidt, and Martin Wegener
}

(Invited Paper)

\begin{abstract}
Photonic metamaterials are man-made materials with "lattice constants" smaller than the wavelength of light. Tailoring the properties of their functional building blocks (atoms) allows one to go beyond the possibilities of usual materials. For example, magnetic dipole moments at optical frequencies $(\mu \neq 1)$ become possible. This aspect substantially enriches the possibilities of optics and photonics and forms the basis for the so-called negativeindex metamaterials. Here, we describe the underlying physics and review the recent progress in this rapidly emerging field.
\end{abstract}

Index Terms-Metamaterial, negative permeability, split-ring resonator (SRR).

\section{INTRODUCTION}

$\mathbf{I}$ $\mathrm{N}$ a usual crystal, the atoms are arranged in a periodic fashion with lattice constants of the order of half a nanometer. This is orders of magnitude smaller than the wavelength of light. For example, green light has a wavelength of about $500 \mathrm{~nm}$. Thus, for a given direction of propagation, the light field experiences an effective homogeneous medium, that is, it does not "see" the underlying periodicity but only the basic symmetries

Manuscript received December 8, 2005; revised June 12, 2006. This work was supported by the Deutsche Forschungsgemeinschaft (DFG) and the State of Baden-Württemberg through the DFG-Center for Functional Nanostructures under Subproject A1.4 and Subproject A1.5. The work of M. Wegener was supported by Project DFG-We 1497/9-1. The work of C. M. Soukoulis was supported by the Alexander von Humboldt Senior-Scientist Award 2002, by the Ames Laboratory under Contract W-7405-Eng-82, by EU FET Project DALHM, and by DARPA under Contract HR0011-05-C-0068.

S. Linden is with the Institut für Nanotechnologie, Forschungszentrum Karlsruhe in der Helmholtz-Gemeinschaft, D-76021 Karlsruhe, Germany.

C. Enkrich, G. Dolling, and M. W. Klein are with the Institut für Angewandte Physik, Universität Karlsruhe (TH), D-76131 Karlsruhe, Germany (e-mail: christian.enkrich@physik.uni-karlsruhe.de).

J. Zhou is with the Department of Electrical and Computer Engineering and the Microelectronics Research Center, Iowa State University, Ames, IA 50011 USA.

T. Koschny is with the Ames Laboratory and the Department of Physics and Astronomy, Iowa State University, Ames, IA 50011 USA, and also with the Institute of Electronic Structure and Laser, Foundation for Research and Technology Hellas, Unversity of Crete, 71110 Heraklion, Crete, Greece.

C. M. Soukoulis is with the Department of Materials Science and Technology, University of Crete, 71110 Heraklion, Crete, Greece, and also with the Institute of Electronic Structure and Laser, Foundation for Research and Technology Hellas, 71110 Heraklion, Crete, Greece, and also with the Ames Laboratory and the Department of Physics and Astronomy, Iowa State University, Ames, IA 50011 USA.

S. Burger and F. Schmidt are with the Zuse Institute Berlin, D-14195 Berlin, Germany, and also with the DFG Forschungszentrum Matheon, D-10623 Berlin, Germany.

M. Wegener is with the Institut für Nanotechnologie, Forschungszentrum Karlsruhe in der Helmholtz-Gemeinschaft, D-76021 Karlsruhe, Germany, and also with the Institut für Angewandte Physik, Universität Karlsruhe (TH), D-76131 Karlsruhe, Germany.

Digital Object Identifier 10.1109/JSTQE.2006.880600 of the crystal. In such materials, the phase velocity of light $c$ may depend on the propagation direction and is generally different from the vacuum speed of light $c_{0}$ by a factor called the refractive index $n=c_{0} / c$ (the slowness). The physical origin are microscopic electric dipoles that are excited by the electric field component of the incoming light and that radiate with a certain retardation. Hence, the electric permittivity is different from unity, i.e., $\epsilon \neq 1$. In contrast to this, magnetic dipoles play no role at optical frequencies in natural substances, i.e., the magnetic permeability is unity, $\mu=1$.

Electromagnetic metamaterials are artificial structures with inter-"atomic" distances (or "lattice constants") that are still smaller than the wavelength of light. Similarly, the light field "sees" an effective homogeneous material for any given propagation direction (quite unlike in a photonic crystal). The building blocks (atoms), however, are not real atoms but are rather made of many actual atoms, often metallic ones. It is this design aspect that allows us to tailor the electromagnetic material properties, in particular the corresponding dispersion relation, to a previously unprecedented degree. For example, it becomes possible to achieve magnetic dipole moments at optical frequencies, i.e., magnetism at optical frequencies $(\mu \neq 1)$. It turns out that, for $\epsilon<0$ and $\mu<0$, the refractive index becomes negative with $n=-\sqrt{\epsilon \mu}<0$ (rather than $n=+\sqrt{\epsilon \mu}>0$ ). This aspect was pointed out by Veselago many years ago [1], but remained an obscurity until rather recently.

In this paper, we first describe the physics of "magnetic atoms" [e.g., the so-called split-ring resonators (SRRs)], which can be best viewed as the magnetic counterpart of the famous Lorentz oscillator model for electric dipoles in optical materials. By simple size scaling, these concepts have recently been brought toward the optical regime. We also discuss the limits of size scaling. Alternative "magnetic atom" designs can push the limits somewhat further and can also ease nanofabrication of these metamaterials.

\section{Physics of SRRs As "Magnetic Atoms"}

It is well known from basic magnetostatics that a magnetic dipole moment can be realized by the circulating ring current of a microscopic coil, which leads to an individual magnetic moment given by the product of the current and the area of the coil. This dipole moment vector is directed perpendicular to the plane of the coil. If such a coil is combined with a plate capacitor, one expects an increased current at a finite-frequency resonance, hence, an increased magnetic dipole moment. Thus, 


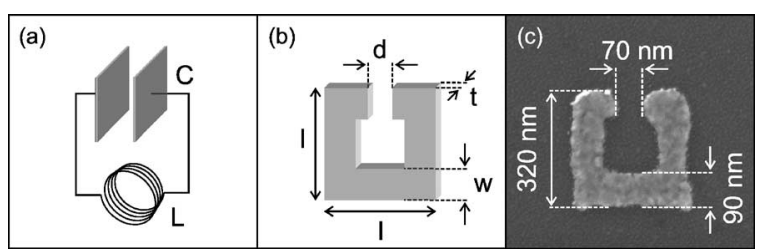

Fig. 1. Illustration of the analogy. (a) A usual $L C$ circuit. (b) SRR. (c) Electron micrograph showing an actually fabricated structure, a gold SRR $(t=20 \mathrm{~nm})$ on a glass substrate. Taken from [6].

a popular design for the magnetic "atoms" is to mimic a usual $L C$ circuit, consisting of a plate capacitor with capacitance $C$ and a magnetic coil with inductance $L$, on a scale much smaller than the relevant wavelength of light.

Fig. 1 shows the analogy of a conventional $L C$ circuit and a metallic SRR on a dielectric surface. The right-hand side (RHS) shows an electron micrograph of a single gold SRR fabricated by standard electron-beam lithography. The name "split-ring resonator" goes back to the work of Hardy and Whitehead in 1981 [2] and that of Pendry et al. in 1999 [3]. This name shall be employed later. However, the SRR has also previously been discussed under the names "slotted-tube resonator" in 1977 [4] in the context of nuclear magnetic resonance (NMR) and "loopgap resonator" in 1996 [5].

\section{A. LC-Resonance Frequency}

The position of the anticipated $L C$-resonance frequency can be estimated by the following crude approach: suppose we can describe the capacitance by the usual textbook formula for a large capacitor with nearby plates $(C \propto$ plate area/distance) and the inductance by the formula for a "long" coil with $N$ windings for $N=1(L \propto$ coil area/length). Using the nomenclature of Fig. 1(b), i.e., the width of the metal $w$, the gap size of the capacitor $d$, the metal thickness $t$, and the width of the coil $l$, we get

$$
C=\epsilon_{0} \epsilon_{C} \frac{w t}{d}
$$

with the relative permittivity of the material in between the plates $\epsilon_{C}$, and

$$
L=\mu_{0} \frac{l^{2}}{t} .
$$

This leads to the eigenfrequency

$$
\omega_{L C}=\frac{1}{\sqrt{L C}}=\frac{1}{l} \frac{c_{0}}{\sqrt{\epsilon_{C}}} \sqrt{\frac{d}{w}} \propto \frac{1}{\text { size }}
$$

and to the $L C$-resonance wavelength

$$
\lambda_{L C}=\frac{2 \pi c_{0}}{\omega_{L C}}=2 \pi l \sqrt{\epsilon_{C}} \sqrt{\frac{w}{d}} \propto \text { size. }
$$

Despite its simplicity and the crudeness of our derivation, this formula contains a lot of correct physics, as confirmed by the numerical calculations (see later): first, it tells us that the $L C$ resonance wavelength is proportional to the linear dimension of the coil $l$, provided that the ratio $w / d$ is fixed. This scaling is valid as long as the metal actually behaves like a metal, i.e., as long as the $L C$-resonance frequency is much smaller than the metal plasma frequency $\omega_{\mathrm{pl}}$. We will describe this fundamental limitation in the following paragraphs. Second, for relevant parameters $\left(\epsilon_{C} \geq 1\right.$ and $\left.w \approx d\right)$, the prefactor is typically of the order of ten, i.e.,

$$
\lambda_{L C} \approx 10 \times l .
$$

Thus, it is possible to arrange these SRRs in the form of an array in the $x y$ plane such that the lattice constant $a_{x y}$ is much smaller than the resonance wavelength, i.e., $a_{x y} \ll \lambda_{L C}$. For example, for a telecommunication wavelength of $\lambda_{L C}=1.5 \mu \mathrm{m}$, the linear dimension of the coil would need to be of the order of $l=150 \mathrm{~nm}$, implying minimum feature sizes around 50 $\mathrm{nm}$ or smaller. Under these conditions, typical values for the capacitance and the inductance are $C \approx 1 \mathrm{aF}$ and $L \approx 1 \mathrm{pH}$, respectively. Third, the dielectric environment influences the resonance via $\epsilon_{C}$, which is, e.g., modified by the presence of a dielectric substrate. Fourth, if one closes the gap, i.e., in the limit $d \rightarrow 0$ or $C \rightarrow \infty$, the resonance wavelength goes to infinity, or equivalently, the resonance frequency $\omega_{L C}$ becomes zero.

\section{B. Limits of Size Scaling}

What are the limits of size scaling according to (3)? This question has been addressed in [7]-[9]: for an ideal metal, i.e., for an infinite electron density $n_{\mathrm{e}}$, hence an infinite metal plasma frequency, a finite current $I$ flowing through the inductance is connected with zero electron velocity, hence, with a vanishing electron kinetic energy. In contrast, for a real metal, i.e., for a finite electron density, the current is inherently connected with a finite electron velocity $v_{\mathrm{e}}$. Thus, one must not only provide the usual magnetic energy $(1 / 2) L I^{2}$ to support the current $I$, but additionally the total electron kinetic energy $N_{\mathrm{e}}\left(m_{\mathrm{e}} / 2\right) v_{\mathrm{e}}^{2}$, where $N_{\mathrm{e}}=n_{\mathrm{e}} V$ is the number of electrons in the SRR contributing to the current. To conveniently incorporate this kinetic energy term into our electromagnetic formulation, we recast it into the form of an additional magnetic energy. Using $n_{\mathrm{e}} e v_{\mathrm{e}}=I / w t$ and the volume (= cross section times the length) of the SRR wire $V=w t(4(l-w)-d)$, we obtain

$$
E_{\text {kin }}=N_{\mathrm{e}} \frac{m_{\mathrm{e}}}{2} v_{\mathrm{e}}^{2}=\frac{1}{2} L_{\mathrm{kin}} I^{2} .
$$

Here, we have introduced the "kinetic inductance"

$$
L_{\text {kin }}=\frac{m_{\mathrm{e}}}{n_{\mathrm{e}} e^{2}} \frac{4(l-w)-d}{w t} \propto \frac{1}{\text { size }} .
$$

While the usual inductance $L$ is proportional to the SRR size [2], the kinetic inductance (7) scales inversely with sizeprovided that all the SRR dimensions are scaled down simultaneously. Thus, the kinetic inductance is totally irrelevant for macroscopic coils but becomes dominant for microscopic inductances, i.e., when approaching the optical frequencies. The kinetic inductance adds to the usual inductance, $L \rightarrow L+L_{\text {kin }}$ in (3), and we immediately obtain the modified scaling for the magnetic resonance frequency

$$
\omega_{L C} \propto \frac{1}{\sqrt{\text { size }^{2}+\text { constant }}} .
$$


Obviously, the magnetic resonance frequency is inversely proportional to the size for a large SRR, whereas it approaches a constant for a small SRR. To evaluate this constant and for the sake of simplicity, we consider the limits $w \ll l, d \ll 4 l$ and the capacitance $C$ according to (1) in air. Inserting the metal plasma frequency $\omega_{\mathrm{pl}}=\sqrt{\left(n_{\mathrm{e}} e^{2}\right) /\left(\epsilon_{0} m_{\mathrm{e}}\right)}$, we obtain the maximum magnetic resonance frequency

$$
\omega_{L C}^{\max }=\sqrt{\frac{1}{L_{\mathrm{kin}} C}}=\omega_{\mathrm{pl}} \sqrt{\frac{d}{4 l}} .
$$

This saturation frequency is further reduced by the dielectric environment and by the skin effect [9], which we have tacitly neglected in our simple reasoning. Furthermore, we remind that our results are implicitly based on the Drude model of the metal intraband transitions. For real metals in the optical regime, the interband transitions also often play a significant role. Our results are meaningful only if $\omega_{L C}^{\max }$ is smaller than the onset frequency of the interband transitions. For example, the interband transitions in aluminum (gold) occur for wavelengths below $800 \mathrm{~nm}(550 \mathrm{~nm})$.

\section{Magnetic Permeability}

One can even obtain an explicit and a simple expression for the magnetic permeability $\mu(\omega)$ from our simple circuit reasoning. We start by considering an excitation configuration where the electric field component of light cannot couple to the SRR (see later), and where the magnetic field is normal to the SRR plane. Under these conditions, the self-induction voltage of the inductance $L$ plus the voltage drop over the capacitance $C$ equals the voltage $U_{\text {ind }}$ induced by the external magnetic field, i.e., $U_{L}+U_{C}=U_{\text {ind }}$ or

$$
L \dot{I}+\frac{1}{C} \int I d t=U_{\text {ind }}=-\dot{\phi}
$$

Again assuming a homogeneous magnetic field in the coil, we obtain the external magnetic flux $\phi=l^{2} \mu_{0} H$, with the external magnetic field $H=H_{0} e^{-i \omega t}+$ c.c. Taking the time derivative of (10) and dividing by $L$ yields

$$
\ddot{I}+\frac{1}{L C} I=\frac{1}{L} \dot{U}_{\text {ind }}=+\omega^{2} \frac{\mu_{0} l^{2}}{L} H_{0} e^{-i \omega t}+\text { c.c. }
$$

Upon inserting the obvious ansatz $I=I_{0} e^{-i \omega t}+$ c.c., we obtain the current $I$, the individual magnetic dipole moment $l^{2} I$, and the magnetization $M=\left(N_{L C} / V\right) l^{2} I$. Here, we have introduced the number of $L C$ circuits $N_{L C}$ per volume $V$. Suppose the lattice constant in the SRR plane is $a_{x y} \geq l$, and $a_{z} \geq t$ in the direction normal to the SRRs. This leads to $N_{L C} / V=1 / a_{x y}^{2} a_{z}$. Finally, using $M=\chi_{\mathrm{m}}(\omega) H, \mu(\omega)=$ $1+\chi_{\mathrm{m}}(\omega)$, and (2) brings us to

$$
\mu(\omega)=1+\frac{\mathcal{F} \omega^{2}}{\omega_{L C}^{2}-\omega^{2}}
$$

Apart from the $\propto \omega^{2}$ numerator, this represents a Lorentz oscillator resonance. Here, we have lumped the various prefactors into the dimensionless quantity $\mathcal{F}$ with

$$
0 \leq \mathcal{F}=\frac{l^{2} t}{a_{x y}^{2} a_{z}} \leq 1
$$

$\mathcal{F}=1$ corresponds to the nearest-neighbor SRRs touching each other-obviously the ultimate upper bound for the accessible SRR density. Thus, we can interpret $\mathcal{F}$ as a filling fraction. Ohmic losses, radiation losses, and other broadening mechanisms can be lumped into a damping $\gamma_{\mathrm{m}}$ of the magnetic resonance.

The bottom line is that the SRR is the magnetic analog of the usual (electric) Lorentz oscillator model. The permeability of the closed ring, i.e., the special case of $d \rightarrow 0 \Rightarrow C \rightarrow \infty \Rightarrow$ $\omega_{L C} \rightarrow 0$ in (12), reduces to

$$
\mu(\omega)=\text { constant }=1-\mathcal{F} \geq 0 .
$$

In other words, the split in the ring is essential for obtaining $\mu(\omega)<0$. For example, for $30 \%$ lateral spacing $\left(a_{x y}=1.3 \times l\right)$ and for a spacing in the vertical direction equal to the SRR thickness $\left(a_{z}=2 \times t\right)$, we obtain $\mathcal{F}=0.30$ and $\mu=0.70$ for closed rings. Note, however, that we have tacitly neglected the interaction among the rings in our considerations leading to this conclusion [10]. The assumption of noninteracting rings is justified for $\mathcal{F} \ll 1$, but becomes questionable for $\mathcal{F} \rightarrow 1$. What qualitative modifications are expected from the interaction of rings? The fringing field of any particular ring at the location of its in-plane neighbors is opposite to its own magnetic dipole moment, hence parallel to the external magnetic field of light. Thus, in-plane interaction tends to effectively increase the value of $\mathcal{F}$ in (14). In contrast, interaction with rings from adjacent parallel planes tends to suppress $\mathcal{F}$ in (14). It is presently unclear, whether a particular arrangement of rings could allow for an increase of $\mathcal{F}$ sufficient to obtain $\mu(\omega)<0$ (also see [3]). Interaction similarly influences the behavior of the split rings.

We note in passing that the description of an isotropic (meta)material in terms of $\epsilon(\omega)$ and $\mu(\omega)$ may be valid, but it is not unique. Indeed, it has already been pointed out in [11] that, alternatively, one can set $\tilde{\mu}=1$ and describe the (meta)material response in terms of the spatial dispersion, i.e., via a wavevector dependence of the electric permittivity $\tilde{\epsilon}(\omega, k)$. One must be aware, however, that the resulting "refractive index" $\tilde{n}(\omega, k)$ looses its usual meaning. A more detailed discussion of this aspect can be found in [12].

Historically, the first demonstration of the negative-index metamaterials was in 2001 at a frequency of about $10 \mathrm{GHz}$ or the wavelength of $3 \mathrm{~cm}$ [13], a regime in which SRR "magnetic atoms" can easily be fabricated on electronic circuit boards. The negative permittivity was achieved by the additional metal stripes. In 2004 [14], $\mu(\omega)<0$ has been demonstrated at a frequency of about $1 \mathrm{THz}(300 \mu \mathrm{m}$ wavelength) using standard microfabrication techniques for the SRR ( [15] reviews this early work).

\section{TOWARD MAGNeTISM AT OptiCAL FREQUenCIES}

At this point, our experimental team entered this field - partly driven by the scepticism that similar materials would not be 
possible at optical frequencies. In our first set of experiments, we scaled down the lateral size of the SRR by two more orders of magnitude, leading to the following parameters: $l=$ $320 \mathrm{~nm}, w=90 \mathrm{~nm}, t=20 \mathrm{~nm}$, and $d=70 \mathrm{~nm}$. On this basis, we anticipated a magnetic resonance at about $\lambda_{L C}=3 \mu \mathrm{m}$. These "magnetic atoms" were arranged on a square lattice with $a_{x y}=450 \mathrm{~nm} \approx 7 \times \lambda_{L C}$ (and larger ones) and a total sample area of $25 \mu \mathrm{m}^{2}$. For normal incidence conditions, however, the light has zero magnetic field component perpendicular to the SRR plane. Thus, excitation via the magnetic field is not possible. Alternatively, the magnetic resonance can also be excited via the electric-field component of light if it has a component normal to the plates of the capacitor, i.e., if the incident light polarization is horizontal. In contrast, for normal incidence and vertical incident polarization, neither the electric nor the magnetic field can couple to the SRR. This "selection rule" can be used to unambiguously identify the magnetic resonance.

\section{A. SRR at Infrared Wavelengths}

Corresponding measured transmittance and reflectance spectra are shown in Fig. 2. Independent of the lattice constant $a_{x y}$, two distinct resonances are clearly visible. With increasing $a_{x y}$, these resonances narrow to some extent because of the reduced interaction between the SRRs, but their spectral position remains essentially unchanged as expected for the electric and magnetic resonant responses of SRRs. This also clearly shows that Bragg diffraction plays no major role. The longwavelength resonance around 3- $\mu \mathrm{m}$ wavelength is present for the horizontal incident polarization and absent for the vertical polarization-as expected from the earlier reasoning. Furthermore, as expected from the previous section, this resonance disappears for the closed rings [Fig. 2(g) and (h)], i.e., for $d \rightarrow 0$, hence $\omega_{L C} \rightarrow 0$. The additional short-wavelength resonance between 1- and 2- $\mu \mathrm{m}$ wavelength is due to the particle plasmon or Mie resonance, mainly exhibiting an electric permittivity, which follows a Lorentz oscillator form according to

$$
\epsilon(\omega)=1+\frac{\mathcal{F} \omega_{\mathrm{pl}}^{2}}{\omega_{\mathrm{Mie}}^{2}-\omega^{2}-i \gamma \omega}
$$

with the metal Drude model damping $\gamma$. The constant $\mathcal{F}$ depends on the SRR volume filling fraction. We will come back to the Mie resonance in more detail later.

All features of the measured spectra (Fig. 2) are reproduced by numerical calculations using a three-dimensional (3-D) finitedifference time-domain approach [6] (not shown here). The corresponding calculated field distributions [6] (not shown here) support the simplistic reasoning on SRRs in the previous section. Retrieving [16] the effective permittivity $\epsilon(\omega)$ and magnetic permeability $\mu(\omega)$ from the calculated spectra, indeed, reveals $\mu<0$ associated with the $\lambda_{L C}=3 \mu \mathrm{m}$ resonance for appropriate polarization conditions [6].

\section{B. SRR at Near-Infrared Wavelengths}

Two questions immediately arise: 1) can the magnetic resonance frequency be further increased by the miniaturization of the SRRs and 2) can one also experimentally demonstrate

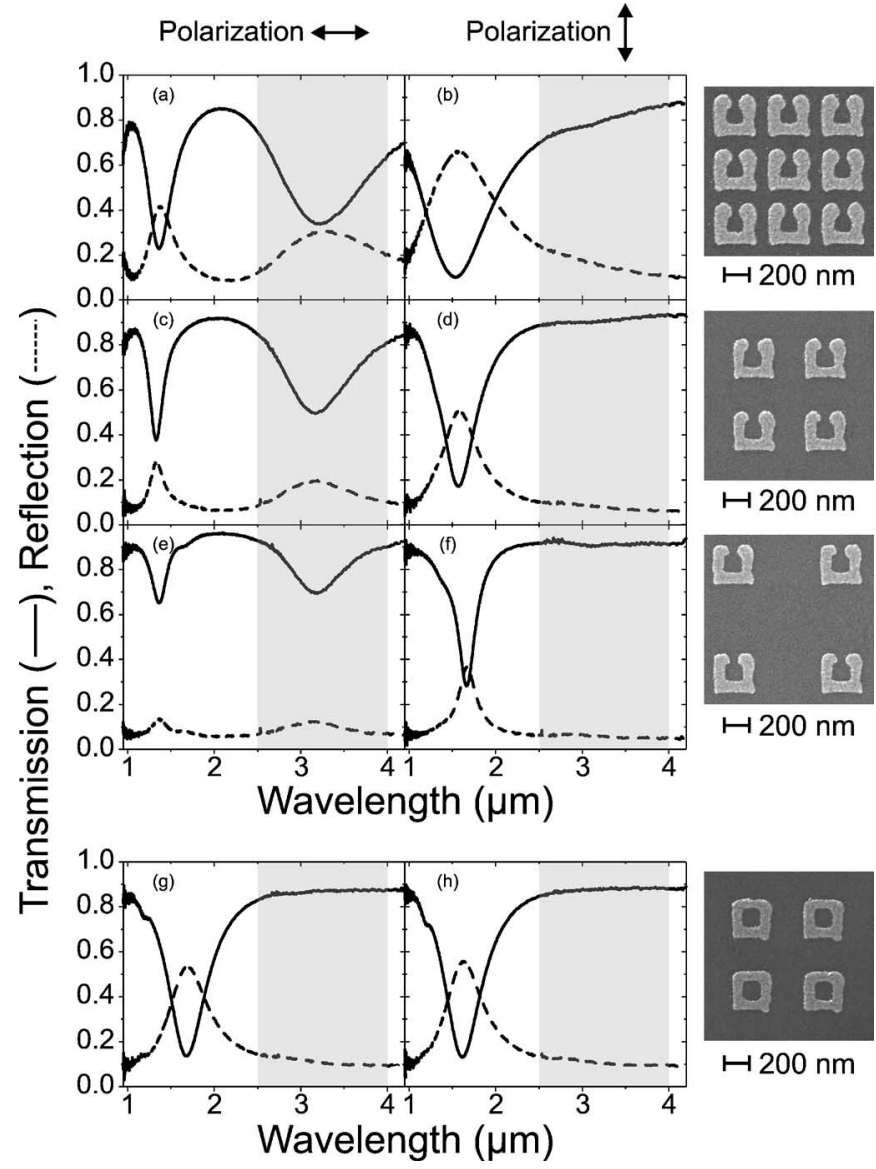

Fig. 2. Measured transmittance and reflectance spectra (normal incidence). In each row of this "matrix," an electron micrograph of the corresponding sample is shown on the RHS. The two polarization configurations are shown on the top of the two columns. (a) and (b) Lattice constant $a=450 \mathrm{~nm}$. (c) and (d) $a=600 \mathrm{~nm}$. (e) and (f) $a=900 \mathrm{~nm}$ correspond to the nominally identical SRRs. (g) and (h) $a=600 \mathrm{~nm}$ correspond to the closed rings. The combination of these spectra unambiguously shows that the resonance at about $3-\mu \mathrm{m}$ wavelength (gray areas) is the $L C$ resonance of the individual SRRs. Taken from [6].

coupling to the magnetic (or $L C$ ) resonance via the magnetic field component of light at optical frequencies? Both aspects have been addressed in our earlier work [17]. Electron micrographs of miniaturized structures are shown in Fig. 3(a). 1) The corresponding measured spectra for horizontal incident polarization in Fig. 3(b) reveal the same (but blue-shifted) resonances as in Fig. 2(a). For vertical incident polarization, compare Fig. 3(c) and Fig. 2(b). 2) In Fig. 4(a), the electric component of the incident light cannot couple to the $L C$ circuit resonance for any angle [in Fig. 4(b) it can]. With increasing angle, however, the magnetic field acquires a component normal to the SRR plane. This component can induce a circulating electric current in the SRR coil via the induction law. This current again leads to the magnetic dipole moment normal to the SRR plane, which can counteract the external magnetic field. The magnitude of this resonance (highlighted by the gray area around $1.5-\mu \mathrm{m}$ wavelength) is indeed consistent with theory [17] (not depicted here), and leads to an effective negative magnetic permeability for propagation in the SRR 


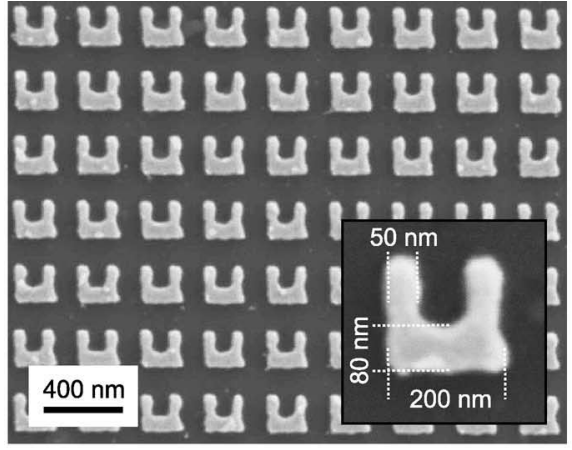

(a)

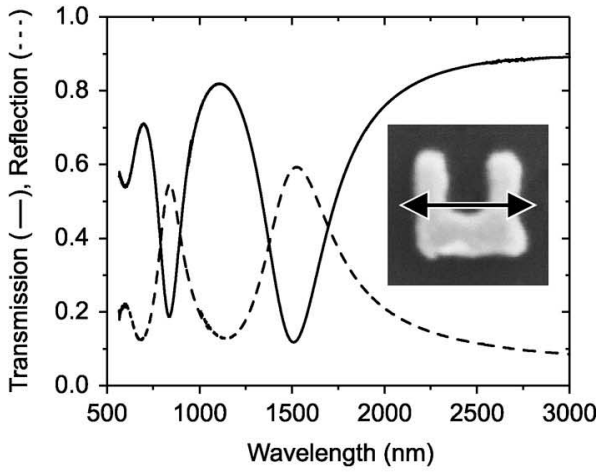

(b)

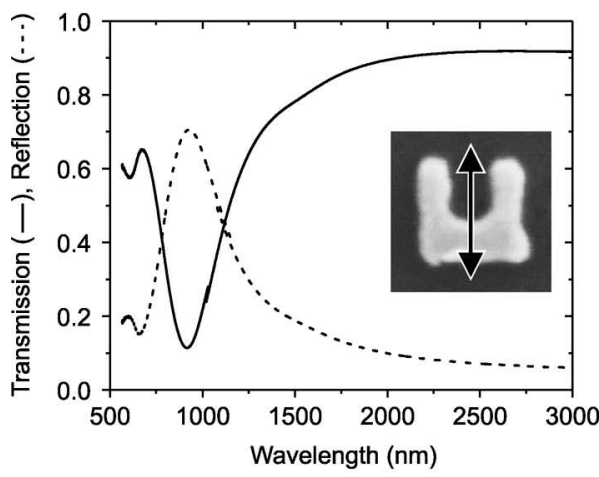

(c)

Fig. 3. (a) Electron micrograph of a split-ring array with a total area of $100 \mu \mathrm{m}^{2}$. Lower RHS inset shows the dimensions of an individual split ring. (b) and (c) Corresponding measured normal-incidence transmittance and reflectance spectra for the horizontal and vertical polarization, respectively. Taken from [17].

plane and for a stack of SRR layers rather than just one layer as considered here. This aspect has been verified explicitly by retrieving the effective permittivity and permeability from the calculated transmittance and reflectance spectra [16], [18].

An unexpected feature of the spectra in Fig. 4(a) is that the 950-nm wavelength Mie resonance at normal incidence splits into two resonances for oblique incidence. This aspect is reproduced by numerical calculations [17], [19]. Intuitively, it can be understood as follows: for normal incidence and vertical polarization, the two similarly shaped vertical SRR arms contribute. These arms are coupled via the SRR's bottom arm (and via the radiation field). As usual, the coupling of the two degenerate modes leads to an avoided crossing with two new effec-

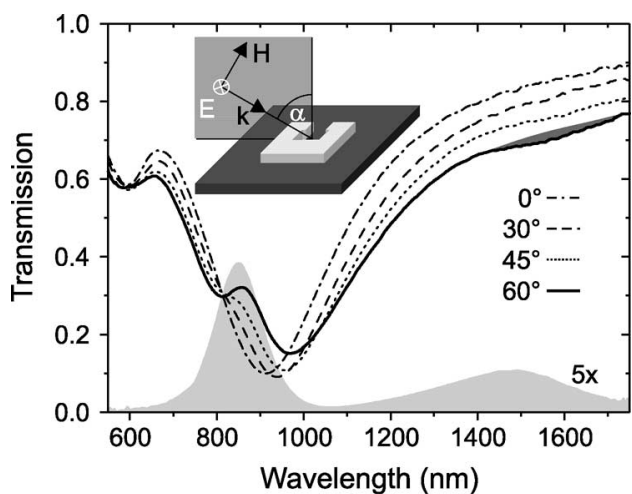

(a)

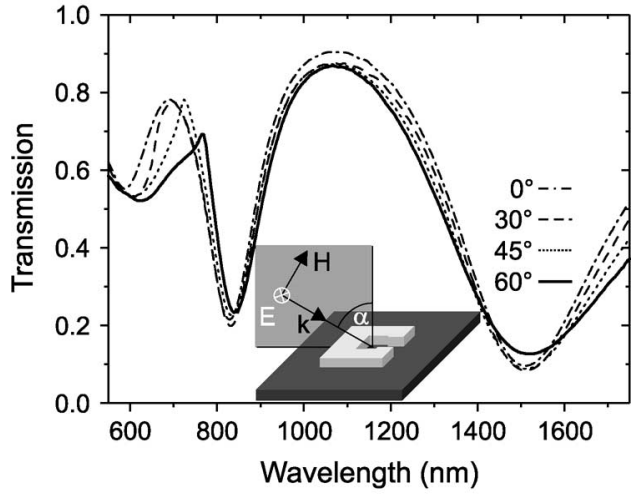

(b)

Fig. 4. Measured transmittance spectra taken for oblique incidence for the configurations shown as insets (where $\alpha=60^{\circ}$ ). (a) Coupling to the fundamental magnetic mode at $1.5-\mu \mathrm{m}$ wavelength is possible only via the magnetic field component of the incident light. (b) Both electric and magnetic fields can couple. Note the small but significant feature in (a) for $60^{\circ}$ around $1.5-\mu \mathrm{m}$ wavelength. The lower gray area in (a) is the transmittance into the linear polarization orthogonal to the incident one for $\alpha=60^{\circ}$. This can be viewed as a fingerprint of the magnetic resonances under these conditions. Taken from [17].

tive oscillation modes, a symmetric and an antisymmetric one, which are frequency down-shifted and up-shifted as compared to the uncoupled resonances, respectively. The antisymmetric mode cannot be excited at all for normal incidence as it has zero effective electric dipole moment. The red-shifted symmetric mode can be excited. It even has a larger effective electric dipole moment than a single arm. Indeed, the Mie resonance for the vertical polarization is deeper and spectrally broader than for the horizontal polarization in Fig. 2, and red-shifted with respect to it. For finite angles of incidence, the phase fronts of the electric field are tilted with respect to the SRR plane. Thus, the vertical SRR arms are excited with a small but finite time delay, equivalent to a finite phase shift. This shift allows coupling to the antisymmetric mode of the coupled system of the two vertical arms as well. In one half cycle of light, one gets a positive charge at the lower left-hand side (LHS) corner of the SRR and a negative charge at the lower RHS corner, resulting in a compensating current in the horizontal bottom arm. Characteristic snapshots of the current distributions in the SRR have been shown schematically in [17].

According to this reasoning for oblique incidence (e.g., $60^{\circ}$ ), we expect a circulating current component for wavelengths near 
the two magnetic resonances at $1.5 \mu \mathrm{m}$ and $800 \mathrm{~nm}$, respectively. Any circulating current is evidently connected with a current in the horizontal bottom arm of the SRR. According to the usual laws of a Hertz dipole, the corresponding charge oscillation in the bottom arm can radiate into the forward direction with an electric field component orthogonal to the incident polarization. In other words, for oblique incidence, the fingerprint of the magnetic resonances is a rotation of polarization. Such rotation is indeed unambiguously observed in the numerical simulations (not shown here, see [17]) and in the experiments [see gray area in Fig. 4(a)].

Further down-scaling of the SRR size is eventually limited by the fact that the resonances stop shifting with decreasing SRR size (see Section II). The shortest magnetic resonance wavelengths that we have achieved are around $900 \mathrm{~nm}$ using gold SRR [20].

We note in passing that there is a continuous transition between the twofold degenerate Mie resonance of a metallic square-shaped "particle" and an SRR, exhibiting a magnetic resonance and a Mie resonance. This transition has been investigated by us [21] using the rapid prototyping capabilities of focused-ion-beam writing.

\section{Cut-Wire Pairs}

The above discussion on the antisymmetric and symmetric eigenmodes of the two coupled vertical SRR arms makes one wonder whether the SRR bottom arm is necessary at all. Indeed, it is not. This has basically been explained earlier and can alternatively be discussed as follows: eliminating the bottom arm can be viewed as introducing a second capacitance into the $L C$ circuit. This effectively reduces the total capacitance in the circuit; hence, it increases the magnetic resonance frequency for a given minimum feature size. However, this eases access to the (near-)visible regime at a reduced fabricational effort. On the other hand, this increased resonance frequency at fixed lattice constant decreases the ratio between the (resonance) wavelength $\lambda_{L C}$ and the lattice constant $a_{x y}$, to about $\lambda_{L C} / a_{x y} \approx 2-3$. In the true metamaterial limit, one aims at $\lambda_{L C} / a_{x y} \gg 1$. Recall that the Bragg condition corresponds to $\lambda_{L C} / a_{x y}=2$. Another significant difference between the original planar SRR design and the resulting cut-wire pairs is that the latter can be rotated by $90^{\circ}$ with respect to the substrate. This not only eases nanofabrication but also allows for the magnetic permeability $\mu(\omega)$ for normal incidence conditions (the magnetic field can be perpendicular to the plane spanned by the two wire pieces, i.e., parallel to the magnetic dipole moment vector). Corresponding theoretical [22]-[25] and experimental [26]-[28] work has been published.

Fig. 5(a)-(c) shows our results for cut-wire pairs of different length but fixed magnesium fluoride $\left(\mathrm{MgF}_{2}\right)$ spacer thickness and fixed gold wire width. The dotted curves in (a) correspond to a nominally identical sample, however, without the upmost gold layer. This single cut-wire sample shows only one pronounced resonance-the Mie resonance-for each polarization. For an incident polarization along the long axis of the cut-wire pairs, however, two resonances are observed that essentially disappear
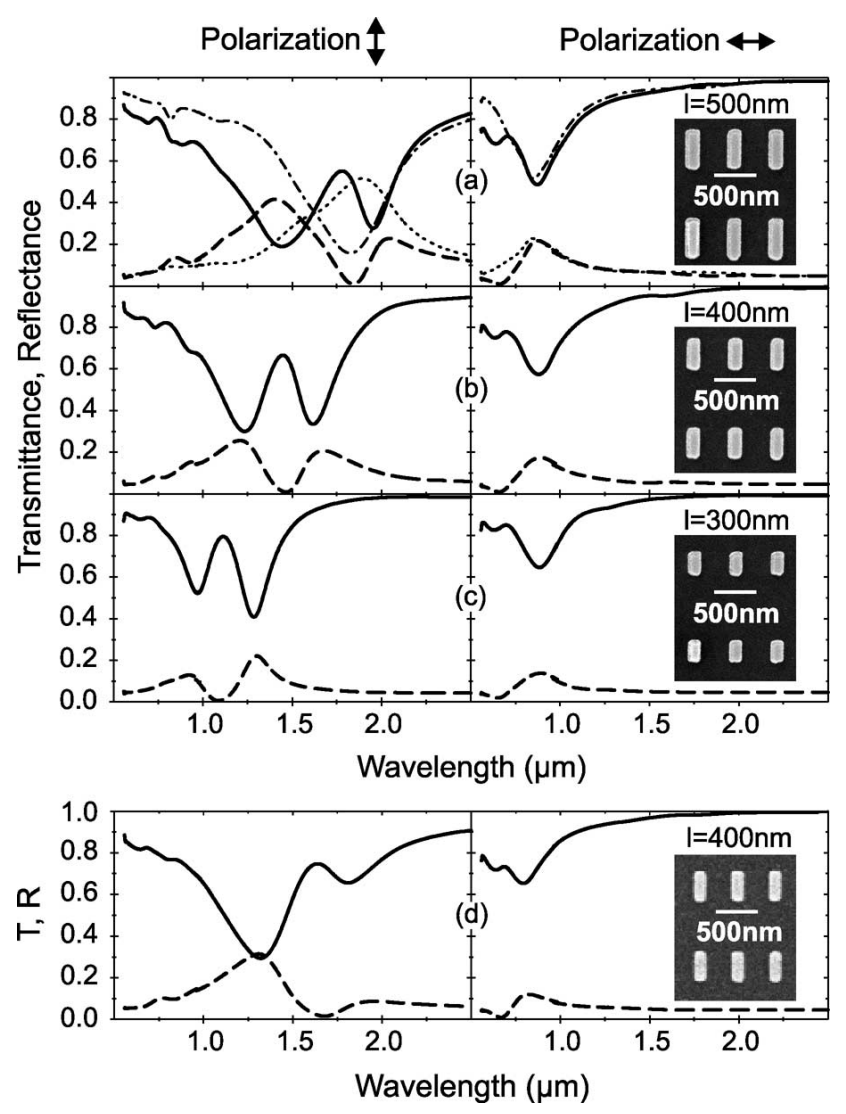

Fig. 5. Measured spectra of transmittance (solid lines) and reflectance (dashed lines) for cut-wire pairs with vertical incident polarization (LHS column) and horizontal polarization (RHS column). Parameters varied: (a) $l=500 \mathrm{~nm}$; (b) $l=400 \mathrm{~nm}$; and (c) $l=300 \mathrm{~nm}$. Fixed parameters for (a)-(c): $\quad w=150 \mathrm{~nm}, t=20 \mathrm{~nm}, d=80 \mathrm{~nm}, a_{x}=500 \mathrm{~nm}, \quad$ and $\quad a_{y}=$ $1050 \mathrm{~nm}$. The dashed-dotted curves in (a) are spectra from a nominally identical structure, but without the upmost gold wire. (d) as (b), but $d=60 \mathrm{~nm}$ rather than $80 \mathrm{~nm}$. The insets in (a)-(d) show corresponding electron micrographs (top view). Taken from [27].

for orthogonal polarization. A comparison of Fig. 5(b) and (d) shows dependence on the $\mathrm{MgF}_{2}$ spacer thickness $d$. As expected from the discussion on the two coupled oscillators, the splitting between the two effective resonances depends on their coupling: For thin (thick) spacers, the coupling is strong (weak); hence, the two resonances are split by a large (small) amount in the spectrum. The obvious polarization dependence of the cut-wire pairs may be undesired in certain cases. Thus, it is also interesting to investigate the samples for which the wire width equals the wire length, i.e., $w=l$. In this case, the cut-wire pairs turn into nanoscopic plate pairs. Their measured optical properties (shown in [27]) are qualitatively similar, yet even more pronounced than in the case of the cut-wire pairs. Reducing the value of $w=l$ allows for the tuning of the resulting resonance positions. The retrieval of $\epsilon(\omega)$ and $\mu(\omega)$ from the calculated spectra corresponding to these parameters yields a negative $\mu(\omega)$ around 1- $\mu \mathrm{m}$ wavelength [27] (not shown). Other groups have even reported a negative real part of the refractive index around 1.5- $\mu \mathrm{m}$ wavelength for the above cut-wire pair structures [28], as deduced from the measured interferometric transmittance and reflectance spectra. 


\section{CONCLUSION}

In contrast to the "conventional textbook wisdom," the magnetic permeability $\mu$ is no longer unity for all the optical materials. While $\mu=1$ holds indeed for all the known natural materials at optical frequencies, for metamaterials $\mu \neq 1$ and even $\mu<0$ can result. In this class of tailored (mostly periodic) structures, SRRs (and variations thereof) play the role of "magnetic atoms" and can lead to the local magnetic dipole moments. Thus, SRRs can be viewed as the magnetic counterparts of the famous Lorentz oscillator model for the electric dipole moments. For the SRRs made from gold, $\mu<0$ can be achieved at telecommunication wavelengths but not in the visible range. Other metals and/or other designs might allow for resonances with $\mu<0$ even in the visible range. Ultimately, the constituent metal plasma frequency sets a fundamental limit.

Where does this field go? Many researchers in the field are driven by the perspective to obtain a negative refractive index $n$ at near infrared or optical frequencies by combining "magnetic atoms" with $\mu<0$ and "electric atoms" with $\epsilon<0$. The search for a negative real part of $n$ itself is motivated by the fascinating possibility of a "perfect lens" [29] providing the subwavelength resolution. Accounting for the "granularity" of the metamaterials and deviations from the strict case of $n=-1$ due to the real [30] and/or the imaginary [31] part of $n$, however, limits the performance of the "perfect lens." Thus, an important parameter is the figure-of-merit (FOM) [32], defined by the negative ratio between the real and imaginary parts of the refractive index $n$ at frequencies where the real part of $n$ is negative. Early experimental work using gold achieved FOM of the order of 1 [33], [34]; more recent experiments employing silver improved this to 3 [35].

Possibly, the real potential of the photonic metamaterials lies in other unexplored areas, for example, in chiral metamaterials or in nonlinear metamaterials. In any case, given today's possibilities regarding the nanofabrication of tailored "atoms," only our own imagination and creativity set the limits.

\section{REFERENCES}

[1] V. G. Veselago, "The electrodynamics of substances with simultaneously negative values of $\epsilon$ and $\mu$," Sov. Phys.-Usp., vol. 10, no. 4, pp. 509-514, 1968.

[2] W. N. Hardy and L. A. Whitehead, "Split-ring resonator for use in magnetic resonance from 200-2000 MHz," Rev. Sci. Instrum., vol. 52, no. 2, pp. 213-216, 1981.

[3] J. B. Pendry, A. J. Holden, D. J. Robbins, and W. J. Stewart, "Magnetism from conductors and enhanced nonlinear phenomena," IEEE Trans. Microw. Theory Tech., vol. 47, no. 14, pp. 2075-2084, Nov. 1999.

[4] H. J. Schneider and P. Dullenkopf, "Slotted tube resonator: A new NMR probe head at high observing frequencies," Rev. Sci. Instrum., vol. 48, no. 1 , pp. 68-73, 1977.

[5] B. T. Ghim, G. A. Rinard, W. Quine, S. S. Eaton, and G. R. Eaton, "Design and fabrication of copper-film loop-gap resonators," J. Magn. Res. A, vol. 120, no. 1, pp. 72-76, 1996.

[6] S. Linden, C. Enkrich, M. Wegener, J. F. Zhou, T. Koschny, and C. M. Soukoulis, "Magnetic response of metamaterials at 100 Terahertz," Science, vol. 306, no. 5700, pp. 1351-1353, 2004.

[7] S. O'Brien and J. B. Pendry, "Magnetic activity at infrared frequencies in structured metallic photonic crystals," J. Phys., Condens. Matter, vol. 14, no. 25 , pp. 6383-6394, 2002.
[8] N.-C. Panoiu and R. M. Osgood, Jr. (2003). Influence of the dispersive properties of metals on the transmission characteristics of lefthanded materials. Phys. Rev. E [Online] 68(1), 016611. Available: http://link.aps.org/abstract/PRE/v68/e016611

[9] J. F. Zhou, T. Koschny, M. Kafesaki, E. N. Economou, J. B. Pendry, and C. M. Soukoulis, "Saturation of the magnetic response of split-ring resonators at optical frequencies," Phys. Rev. Lett., vol. 95, p. 223902, 2005.

[10] M. Gorkunov, M. Lapine, E. Shamonina, and K. H. Ringhofer. (2002). Effective magnetic properties of a composite material with circular conductive elements. Eur. Phys. J. B [Online] 28(3), pp. 263-269. Available: http://dx.doi.org/10.1140/epjb/e2002-00228-4

[11] L. D. Landau and E. L. Lifshitz, Electrodynamics of Continuous Media, 2nd ed. New York: Pergamon, 1960.

[12] K. Busch, G. von Freymann, S. Linden, L. Tkeshelashvili, S. Mingaleev, and M. Wegener, "Periodic nanostructures for photonics," Phys. Rep., submitted for publication.

[13] R. A. Shelby, D. R. Smith, and S. Schultz, "Experimental verification of a negative index of refraction," Science, vol. 292, no. 5514, pp. 77-79, 2001.

[14] T. J. Yen, W. J. Padilla, N. Fang, D. C. Vier, D. R. Smith, J. B. Pendry, D. N. Basov, and X. Zhang, "Terahertz magnetic response from artificial materials," Science, vol. 303, no. 5663, pp. 1494-1496, 2004.

[15] D. R. Smith, J. B. Pendry, and M. C. K. Wiltshire, "Metamaterials and negative refractive index," Science, vol. 305, no. 5685, pp. 788-792, 2004.

[16] D. R. Smith, S. Schultz, P. Markos, and C. M. Soukoulis. (2002). Determination of effective permittivity and permeability of metamaterials from reflection and transmission coefficients. Phys. Rev. B [Online] 65(19), p. 195104. Available: http://link.aps.org/abstract/PRB/v65/e195104

[17] C. Enkrich, M. Wegener, S. Linden, S. Burger, L. Zschiedrich, F. Schmidt, J. F. Zhou, T. Koschny, and C. M. Soukoulis, "Magnetic metamaterials at telecommunication and visible frequencies," Phys. Rev. Lett., vol. 95, no. 20, p. 203901, 2005.

[18] T. Koschny, P. Markos, E. N. Economou, D. R. Smith, D. C. Vier, and C. M. Soukoulis. (2005). Impact of the inherent periodic structure on the effective medium description of left-handed and related meta-materials. Phys. Rev. B [Online] 71(24), p. 245105. Available: http://link.aps.org/abstract/PRB/v71/e245105

[19] S. Burger, L. Zschiedrich, R. Klose, A. Schädle, F. Schmidt, C. Enkrich, S. Linden, M. Wegener, and C. M. Soukoulis, "Numerical investigation of light scattering off split-ring resonators," in Proc. SPIE, 2005, vol. 5955, pp. 18-26.

[20] M. W. Klein, C. Enkrich, M. Wegener, C. M. Soukoulis, and S. Linden. (2006). Single-slit split-ring resonators at optical frequencies: Limits of size scaling. Opt. Lett. [Online] 31(9), pp. 1259-1261. Available: http://www.opticsinfobase.org/abstract.cfm?URI=ol-31-9-1259.

[21] C. Enkrich, F. Pérez-Willard, D. Gerthsen, J. F. Zhou, C. M. Soukoulis, M. Wegener, and S. Linden, "Focused-ion-beam nanofabrication of nearinfrared magnetic metamaterials," Adv. Mater., vol. 17, no. 21, pp. $2547-$ 2549, 2005.

[22] A. N. Lagarkov and A. K. Sarychev, "Electromagnetic properties of composites containing elongated conducting inclusions," Phys. Rev. B, vol. 53, no. 10 , pp. 6318-6336, 1996.

[23] L. Panina, A. Grigorenko, and D. Makhnovskiy, "Optomagnetic composite medium with conducting nanoelements," Phys. Rev. B, vol. 66, no. 15, p. 155411, 2002.

[24] V. A. Podolskiy, A. K. Sarychev, and V. M. Shalaev, "Plasmon modes in metal nanowires and left-handed materials," J. Nonlinear Opt. Phys. Mater., vol. 11, no. 1, pp. 65-74, 2002.

[25] V. A. Podolskiy, A. K. Sarychev, E. E. Narimanov, and V. M. Shalaev, "Resonant light interaction with plasmonic nanowire systems," J. Opt.A, Pure Appl. Opt., vol. 7, no. 2, pp. S32-S37, 2005.

[26] A. N. Grigorenko, A. K. Geim, H. F. Gleeson, Y. Zhang, A. A. Firsov, I. Y. Khrushchev, and J. Petrovic, "Nanofabricated media with negative permeability at visible frequencies," Nature, vol. 438, no. 7066, pp. 335338, 2005.

[27] G. Dolling, C. Enkrich, M. Wegener, J. F. Zhou, C. M. Soukoulis, and S. Linden. (2005). Cut-wire pairs and plate pairs as magnetic atoms for optical metamaterials. Opt. Lett. [Online] 30(23), pp. 3198-3200. Available: http://www.opticsinfobase.org/abstract.cfm?URI=ol-30-23-3198

[28] V. P. Drachev, W. Cai, U. Chettiar, H.-K. Yuan, A. K. Sarychev, A. V. Kildishev, G. Klimeck, and V. M. Shalaev. (2006). Experimental verification of an optical negative-index material. Laser Phys. Lett. [Online] 3(1), pp. 49-55. Available: http://dx.doi.org/10.1002/lapl. 200510062 
[29] J. B. Pendry. (2000). Negative refraction makes a perfect lens. Phys. Rev. Lett. [Online] 85(18), pp. 3966-3969. Available: http://link.aps.org/abstract/PRL/v85/p3966

[30] R. Merlin, "Analytical solution of the almost-perfect-lens problem," Appl. Phys. Lett., vol. 84, no. 8, pp. 1290-1292, 2004.

[31] V. A. Podolskiy and E. E. Narimanov, "Near-sighted superlens," Opt Lett., vol. 30, no. 1, pp. 75-77, 2005.

[32] S. Zhang, W. Fan, K. J. Malloy, S. R. Brueck, N. C. Panoiu, and R. M. Osgood, "Near-infrared double negative metamaterials," Opt. Express, vol. 13, no. 13, pp. 4922-4930, 2005

[33] S. Zhang, W. Fan, N. C. Panoiu, K. J. Malloy, R. M. Osgood, and S. R. J. Brueck. (2005). Experimental demonstration of near-infrared negative-index metamaterials. Phys. Rev. Lett. [Online] 95(13), p. 137404 Available: http://link.aps.org/abstract/PRL/v95/e137404

[34] G. Dolling, C. Enkrich, M. Wegener, C. M. Soukoulis, and S. Linden, "Simultaneous negative phase and group velocity of light in a metamaterial," Science, vol. 312, no. 5775, pp. 892-894, 2006.

[35] - (2006). Low-loss negative-index metamaterial at telekommunication wavelengths. Opt. Lett. [Online] 31(12), 1800-1802. Available: http://www.opticsinfobase.org/abstract.cfm?URI=ol-31-12-1800

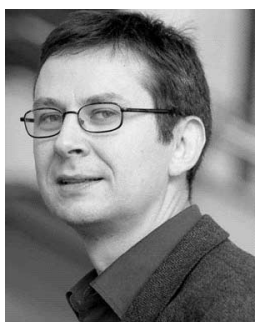

Stefan Linden received the Ph.D. degree in physics from the Universität Marburg, Marburg, Germany, in 2002

He received the Feodor Lynen Research Fellowship from the Alexander von Humboldt Foundation and spent one year at the University of Toronto working on the nonlinear properties of photonic crystals. He is currently a Postdoctoral Researcher at the Institut für Nanotechnologie, Forschungszentrum Karlsruhe, Karlsruhe, Germany. He also leads a Helmholtz Young Investigators Group. He has a strong background in ultrafast spectroscopy, optics of metal nanostructures, photonic crystals, and electron-beam lithography. His current research interests include the nonlinear optical properties of photonic crystals and the realization of left-handed metamaterials for telecommunication wavelengths.

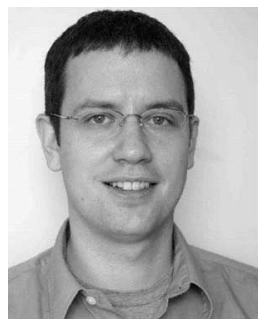

Christian Enkrich received the Ph.D. degree in physics from the Universität Karlsruhe (TH), Karlsruhe, Germany, in 2006.

He is currently with the Institut für Angewandte Physik, Universität Karlsruhe (TH). His current research interests include fabrication and characterization of metamaterials for the near-infrared wavelength regime and corresponding numerical simulations.

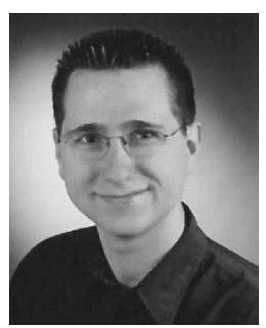

Gunnar Dolling was born in Germany in 1982. He received the Diploma in physics from the Universität Karlsruhe (TH), Karlsruhe, Germany, in 2006, where he is currently working toward the Ph.D. degree in physics, focusing on negative-index metamaterials at telecommunication and visible wavelengths.

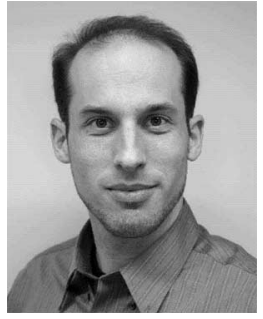

Matthias W. Klein received the Diploma in physics from the Universität Heidelberg, Heidelberg, Germany, in 2002. He is currently working toward the Ph.D. degree in physics at the Universität Karlsruhe (TH), Karlsruhe, Germany.

During 1999-2000, he was a Cornell-Heidelberg Exchange Fellow at Cornell University, Ithaca, NY, where he was engaged in experimental and theoretical research in organic nanoelectronics. He performed his diploma thesis in the field of quantum optics. His current research interests include the area of nanophotonics, with focus on linear and nonlinear experiments with metallic photonic crystals and metamaterials.

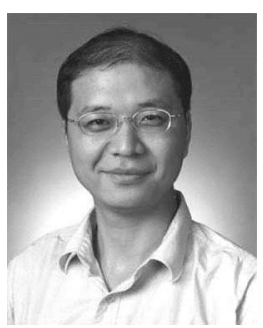

Jiangfeng Zhou was born in 1976. He received the M.S. degree in physics from Beijing University, Beijing, China, in 2001. He is currently working toward the Ph.D. degree in electrical engineering at Iowa State University, Ames.

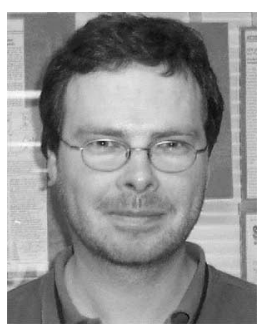

Thomas Koschny was born in 1971 . He received the M.Sc. and Ph.D. degrees from the Universität Leipzig, Leipzig, Germany, in 1997 and 2001, respectively, both in physics.

During 2000-2002, he was a Postdoctoral Researcher of quantum Hall effect at the PhysikalischTechnische Bundesanstalt Braunschweig, Germany. Since 2003, he has been working on the electromagnetic wave propagation in left-handed metamaterials at the Institute of Electronic Structure and Laser, Foundation for Research and Technology Hellas, Crete, Greece. Since 2004, he has been a Researcher at the Ames Laboratory and the Department of Physics and Astronomy at Iowa State University, Ames. His current research interests include the theory of left-handed metamaterials and photonic crystals.

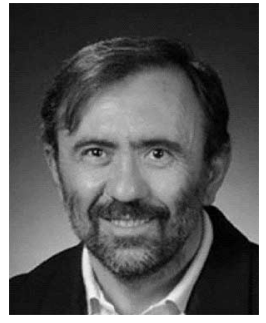

Costas M. Soukoulis received the B.S. degree from the University of Athens, Athens, Greece, and the Ph.D. degree from the University of Chicago, Chicago, IL, in 1974 and 1978, respectively, both in physics.

From 1978 to 1981 , he was a Visiting Assistant Professor in the Physics Department, University of Virginia. During 1981-1984, he was with Exxon Research and Engineering Company. Since 1984, he has been at Iowa State University and the Ames Laboratory, Ames, IA, where he is currently a Distinguished Professor of physics. Since 1983, he has been an Associate Faculty Member of the Foundation for Research and Technology Hellas, Crete, Greece, and since 2001, he has been a Professor (part-time) in the Department of Materials Science and Engineering, University of Crete, Crete. He is the author or coauthor of approximately 300 publications and 7000 citations. He is the holder of two patents for photonic crystals. His current research interests include photonic crystals, left-handed materials, random lasers, light localizations, and theory of disordered systems.

Prof. Soukoulis is a Fellow of the American Physical Society, Optical Society of America, and American Association for the Advancement of Science. He is the Senior Editor of Photonic Nanostructures: Fundamentals and Applications. 


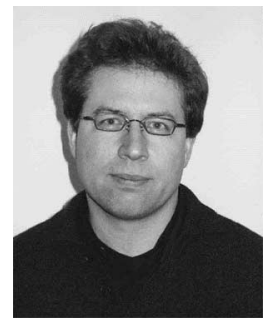

Sven Burger received the Ph.D. degree in physics from the University of Hannover, Hannover, Germany, in 2000 .

He was a Faculty Member at the Institute of Quantum Optics, University of Hannover. From 2000 to 2001, he was a Postdoctoral Researcher in an EU TMR network at the European Laboratory for Nonlinear Spectroscopy, Florence, Italy. Since 2002, he has been with the Numerical Analysis and Modeling Group, Zuse Institute Berlin, Berlin, Germany. He is also with the DFG Research Center Matheon, Berlin, and with the spin-off company JCMwave. He has been engaged in research on solitons in Bose-Einstein condensates and the realization of arrays of Josephson junctions in atomic systems. His current research interests include developing numerical methods for Maxwell's equations, with applications to photonic crystals, photolithography, metamaterials, among others.

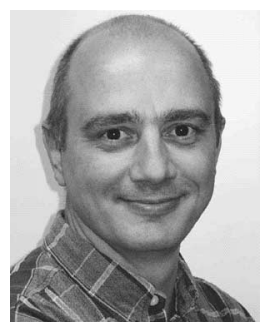

Frank Schmidt received the Ph.D. degree in electrical engineering/optical telecommunications from Humboldt University, Berlin, Germany, in 1989, and the Habilitation (mathematics) from the Free University Berlin, Berlin, in 2002.

Since 1992, he has been a Researcher at the Zuse Institute Berlin, Berlin, where he currently heads the Computational Nano-Optics Group. He is one of the Scientists-in-Charge of Electronic Circuits and Optical Technologies of the DFG Research Center Matheon, Berlin, and is one of the founders of the spin-off company JCMwave. He is the author or coauthor of about 40 papers in the field of numerical methods in optics.

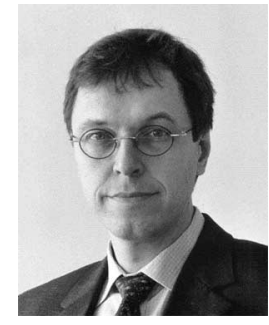

Martin Wegener received the Ph.D. degree in physics from Johann Wolfgang Goethe Universität Frankfurt, Frankfurt, Germany, in December 1987.

After being a Postdoctoral Fellow at AT\&T Bell Laboratories, Holmdel, NJ, he became an Associate Professor at the Universität Dortmund in June 1990. Since October 1995, he has been a Full Professor at the Universität Karlsruhe (TH), Karlsruhe, Germany. Since 1997, he has been the Head of the DFG Graduate School Collective Phenomena in Solids. Since 2001, he has been a Group Leader at the Institut für Nanotechnologie, Forschungszentrum Karlsruhe, and since 2001, a Coordinator of the Karlsruhe DFG-Center for Functional Nanostructures. He is the author of two textbooks, a coauthor of about 150 publications in peer-reviewed journals and international conference proceedings, and has given about 50 invited talks at international conferences. His current research interests include different areas of photonics, such as, nonlinear-optical spectroscopy, ultrafast spectroscopy, extreme nonlinear optics, near-field optical spectroscopy, photonic bandgap materials, and photonic metamaterials.

Dr. Wegener serves as Referee for a number of national committees and various international journals. Since 2005, he has been a Topical Editor of the Journal of the Optical Society of America. He was the recipient of the Research Award of the Alfried Krupp von Bohlen und Halbach-Stiftung in 1993, the Teaching Award of the State of Baden-Württemberg in 1998, the DFG-Leibniz Award in 2000, and the Descartes Prize of the European Union in 2005 